\title{
COHOMOLOGY GROUPS OF SECTIONS OF HOMOGENEOUS LINE BUNDLES OVER A TOROIDAL GROUP
}

\author{
Yukitaka ABE
}

(Received 21 July 2015)

\begin{abstract}
We completely determine cohomology groups of sections of homogeneous line bundles over a toroidal group.
\end{abstract}

\section{Introduction}

A toroidal group is a connected complex Lie group without non-constant holomorphic functions. Such a group appears as the steinizer of a complex Lie group $[\mathbf{8 , 9}$ ]. It is well known that a toroidal group is commutative. Then it is isomorphic to a quotient group $\mathbb{C}^{n} / \Gamma$ of $\mathbb{C}^{n}$ by a discrete subgroup $\Gamma$. A complex torus is a compact toroidal group. By the RemmertMorimoto theorem [7, 9] every connected commutative complex Lie group is isomorphic to the product of copies of $\mathbb{C}$, copies of $\mathbb{C}^{*}=\mathbb{C} \backslash\{0\}$ and a toroidal group. A toroidal group plays an important role in the study of complex Lie groups. Moreover, the relations to the number theory are known (cf. $[2,3])$.

Let $X$ be a toroidal group. The cohomology groups $H^{p}(X, \mathcal{O})(p \geq 1)$ were completely determined by Kazama [5]. The next problem is to determine $H^{p}(X, \mathcal{O}(L))$ for any holomorphic line bundle $L$ over $X$. If $X$ is compact, i.e., a complex torus, then we know the cohomology groups $H^{p}(X, \mathcal{O}(L))$ for any $L$. The general result is known as the Index theorem. In this case we need tools which are valid for compact Kähler manifolds. Unfortunately, they are not applicable to non-compact toroidal groups.

In this paper we consider homogeneous line bundles $L$ over a toroidal group $X=\mathbb{C}^{n} / \Gamma$ with rank $\Gamma=n+m$. It is known that $X$ has the structure of a principal $\left(\mathbb{C}^{*}\right)^{n-m}$-bundle $\mu: X \longrightarrow \mathbb{T}$ over an $m$-dimensional complex torus $\mathbb{T}$. We determine the cohomology groups $H^{p}(X, \mathcal{O}(L))$ for $p \geq 1$. The following three cases occur when $L$ is not analytically trivial:

(1) $H^{p}(X, \mathcal{O}(L))=0$ for $p \geq 1$;

(2) $H^{p}(X, \mathcal{O}(L)) \cong H^{p}(\mathbb{T}, \mathcal{O})$ for $p \geq 1$;

(3) $\quad H^{p}(X, \mathcal{O}(L))$ is a non-Hausdorff Fréchet space, then, of infinite dimension for $1 \leq$ $p \leq m$

(Theorem 9.1). It seems to us that this result is the first step beyond the case of $H^{p}(X, \mathcal{O})$. When $X$ is a complex torus, we know $H^{p}(X, \mathcal{O}(L))=0(p \geq 1)$ for any homogeneous line bundle $L$ over $X$ which is not analytically trivial. Our method gives another proof of this fact. We give examples which show that each of the above cases really occurs.

2010 Mathematics Subject Classification: Primary 32L10; Secondary 32M05.

Keywords: cohomology groups; homogeneous line bundles; toroidal groups.

(C) 2016 Faculty of Mathematics, Kyushu University 
The paper is organized as follows. In Section 2 we state standard coordinates and real coordinates of $\mathbb{C}^{n}$ and the relation between them. In Section 3 we collect some facts about homogeneous line bundles. In Section 4 we introduce sheaves $\mathcal{F}^{r, s}$ and $\mathcal{F}^{r, s}(L)$, and give Dolbeault-Kazama isomorphisms. Every $\Gamma$-periodic $C^{\infty}$ function on $\mathbb{C}^{n}$ which is holomorphic with respect to the last $n-m$ variables has the Fourier expansion. In Section 5 we explain the properties of such Fourier expansions and their derivatives. The spaces $H^{0}\left(X, \mathcal{F}^{r, s}\right)$ and $H^{0}\left(X, \mathcal{F}^{r, s}(L)\right)$ are isomorphic as Fréchet spaces. Using these isomorphisms, we translate $\bar{\partial}$-equations to certain equations of $\Gamma$-periodic differential forms in Section 6. In Section 7 we obtain formal solutions of the above equations. Then it suffices to consider the convergence of formal solutions. We give conditions for the convergence in Section 8. Finally, we prove the main result in Section 9. In the last section we construct examples.

\section{Preliminaries}

Let $X=\mathbb{C}^{n} / \Gamma$ be a toroidal group with $\operatorname{rank} \Gamma=n+m$. We use standard coordinates $z=\left(z_{1}, \ldots, z_{n}\right)$ of $\mathbb{C}^{n}$ for $X$ and a period matrix $P$ of $X$ in the first normal form as

$$
P=\left(\begin{array}{ll}
I_{n} & S
\end{array}\right), \quad S=\left(\begin{array}{l}
S_{1} \\
S_{2}
\end{array}\right),
$$

where $I_{n}$ is the unit matrix of degree $n$ and $S$ is a complex $(n, m)$-matrix with $\operatorname{det}\left(\operatorname{Im}\left(S_{1}\right)\right) \neq$ 0 . The matrix $S$ satisfies the irrationality condition

(IS) for any $\tau=\left(\tau_{1}, \ldots, \tau_{n}\right) \in \mathbb{Z}^{n} \backslash\{0\}$ we have $\tau S \notin \mathbb{Z}^{m}$

in standard coordinates because $X$ is a toroidal group (cf. [4]). We write $\left(I_{n} \quad S\right)=$ $\left(e_{1}, \ldots, e_{n}, s_{1}, \ldots, s_{m}\right)$. For $j=m+1, \ldots, n$ we set $s_{j}:=\sqrt{-1} e_{j}$. Then $\left\{e_{1}, \ldots, e_{n}\right.$, $\left.s_{1}, \ldots, s_{n}\right\}$ is a basis of $\mathbb{C}^{n}$ over $\mathbb{R}$. We take real coordinates $t=\left(t_{1}, \ldots, t_{2 n}\right)$ of $\mathbb{C}^{n}$ defined by

$$
\sum_{i=1}^{n} z_{i} e_{i}=\sum_{i=1}^{n} t_{i} e_{i}+\sum_{i=1}^{n} t_{n+i} s_{i}
$$

A column vector $s_{j}$ is written as $s_{j}={ }^{\mathrm{t}}\left(s_{1 j}, \ldots, s_{n j}\right)$. We set $A:=\left(a_{i j}\right)_{1 \leq i, j \leq n}$ and $B:=$ $\left(b_{i j}\right)_{1 \leq i, j \leq n}$, where $a_{i j}:=\operatorname{Re}\left(s_{i j}\right)$ and $b_{i j}:=\operatorname{Im}\left(s_{i j}\right)$. We note that $a_{i j}=0$ if $1 \leq i \leq n$ and $m+1 \leq j \leq n$. Let $C=\left(c_{i j}\right)_{1 \leq i, j \leq n}:=B^{-1}$. Then we have $c_{i j}=\delta_{i j}$ for $1 \leq i \leq n$ and $m+1 \leq j \leq n$. We write $z_{i}=x_{i}+\sqrt{-1} y_{i}, i=1, \ldots, n$ as usual. Then we have the relations

$$
t_{j}=x_{j}-\sum_{k=1}^{n}\left(\sum_{\ell=1}^{n} a_{j \ell} c_{\ell k}\right) y_{k} \quad \text { and } \quad t_{n+j}=\sum_{k=1}^{n} c_{j k} y_{k}
$$

for $j=1, \ldots, n$. Therefore we obtain

$$
\frac{\partial}{\partial \bar{z}_{j}}=\frac{1}{2}\left\{\frac{\partial}{\partial t_{j}}+\sqrt{-1}\left(-\sum_{k=1}^{n} \sum_{\ell=1}^{n} a_{k \ell} c_{\ell j} \frac{\partial}{\partial t_{k}}+\sum_{k=1}^{n} c_{k j} \frac{\partial}{\partial t_{n+k}}\right)\right\}
$$

for $j=1, \ldots, n$.

Let $\mathbb{T}=\mathbb{C}^{m} / \Lambda$ be an $m$-dimensional complex torus, where $\Lambda$ is a discrete subgroup of $\mathbb{C}^{m}$ with period matrix $\left(\begin{array}{ll}I_{m} & S_{1}\end{array}\right)$. The toroidal group $X$ has the structure of a principal $\left(\mathbb{C}^{*}\right)^{n-m}$-bundle $\mu: X \longrightarrow \mathbb{T}$ over $\mathbb{T}$ by the projection $\left(z_{1}, \ldots, z_{n}\right) \longmapsto\left(z_{1}, \ldots, z_{m}\right)$. 


\section{Homogeneous line bundles}

For any $x$ in a toroidal group $X=\mathbb{C}^{n} / \Gamma$ we define a translation $T_{x}: X \longrightarrow X, y \mapsto y+x$.

Definition 3.1. A holomorphic line bundle $L$ over $X$ is said to be homogeneous if $L$ and $T_{x}^{*} L$ is analytically isomorphic for any $x \in X$.

A homomorphism $\rho: \Gamma \longrightarrow \mathbb{C}^{*}$ is called a (one-dimensional) representation of $\Gamma$. Since it is considered as a factor of automorphy, it defines a holomorphic line bundle over $X$. It is obvious by definition that if $L$ is a holomorphic line bundle given by a representation of $\Gamma$, then it is homogeneous. When $X$ is a complex torus, it is well known that for a holomorphic line bundle $L$ over $X$ the following statements are equivalent:

(1) $L$ is topologically trivial;

(2) $L$ is given by a representation of $\Gamma$;

(3) $L$ is homogeneous.

The above equivalence does not hold for a toroidal group in general. However, we have the following proposition.

PROPOSITION 3.2. (Abe [1]) Let L be a holomorphic line bundle over a toroidal group $X=\mathbb{C}^{n} / \Gamma$. Then $L$ is homogeneous if and only if it is given by a representation of $\Gamma$.

Throughout this paper we assume that $L$ is a homogeneous line bundle over a toroidal group $X=\mathbb{C}^{n} / \Gamma$ given by a representation $\rho: \Gamma \longrightarrow \mathbb{C}^{*}$ of $\Gamma$. Then there exists a homomorphism $d: \Gamma \longrightarrow \mathbb{C}$ such that $\rho(\gamma)=\mathbf{e}(d(\gamma))(\gamma \in \Gamma)$, where $\mathbf{e}(*)=$ $\exp (2 \pi \sqrt{-1} *)$.

LEMMA 3.3. The homomorphism $d: \Gamma \longrightarrow \mathbb{C}$ is equivalent to a homomorphism $\tilde{d}: \Gamma \longrightarrow$ $\mathbb{R}$ with $\tilde{d}\left(e_{m+j}\right)=0$ for $j=1, \ldots, n-m$ as summands of automorphy.

Proof. First we may assume $d\left(e_{j}\right)=0$ for $j=1, \ldots, n$ because $\rho=\mathbf{e}(d)$ gives a topologically trivial holomorphic line bundle (cf. [10, p. 201, Proposition 7]). We define a discrete subgroup $\widetilde{\Gamma}$ of rank $2 n$ by

$$
\widetilde{\Gamma}:=\Gamma \oplus \bigoplus_{j=1}^{n-m} \mathbb{Z} s_{m+j} .
$$

Putting $d\left(s_{m+j}\right)=0$ for $j=1, \ldots, n-m$, we extend $d$ to a homomorphism $d: \widetilde{\Gamma} \longrightarrow \mathbb{C}$. Furthermore, it is extended to an $\mathbb{R}$-linear mapping $d: \mathbb{C}^{n} \longrightarrow \mathbb{C}$. If we set $k:=\operatorname{Im}(d)$, then $k: \mathbb{C}^{n} \longrightarrow \mathbb{R}$ is an $\mathbb{R}$-linear mapping such that

$$
k\left(e_{j}\right)=0, \quad j=1, \ldots, n
$$

and

$$
k\left(s_{m+j}\right)=k\left(\sqrt{-1} e_{m+j}\right)=0, \quad j=1, \ldots, n-m .
$$

We define a $\mathbb{C}$-linear mapping $\ell: \mathbb{C}^{n} \longrightarrow \mathbb{C}$ by

$$
\ell(v):=k(\sqrt{-1} v)+\sqrt{-1} k(v), \quad v \in \mathbb{C}^{n} .
$$

Set $\tilde{d}(\gamma):=d(\gamma)-\ell(\gamma)$ for $\gamma \in \Gamma$. Then $\tilde{d}: \Gamma \longrightarrow \mathbb{R}$ is a homomorphism satisfying $\tilde{d}\left(e_{m+j}\right)=0$ for $j=1, \ldots, n-m$. Since $\ell$ is $\mathbb{C}$-linear, $d$ and $\tilde{d}$ are equivalent.

From now on we assume that the representation $\rho=\mathbf{e}(d)$ is given by a homomorphism $d: \Gamma \longrightarrow \mathbb{R}$ having the properties stated in Lemma 3.3. 


\section{Dolbeault-Kazama isomorphisms}

Consider the structure of a principal $\left(\mathbb{C}^{*}\right)^{n-m}$-bundle $\mu: X \longrightarrow \mathbb{T}$ stated in Section 2 . We write standard coordinates $z=\left(z_{1}, \ldots, z_{n}\right)$ as $z=\left(z^{\prime}, z^{\prime \prime}\right)$, where $z^{\prime}=\left(z_{1}, \ldots, z_{m}\right)$ and $z^{\prime \prime}=\left(z_{m+1}, \ldots, z_{n}\right)$. The $\bar{\partial}$-operator is decomposed as $\bar{\partial}=\bar{\partial}_{1}+\bar{\partial}_{2}$, where $\bar{\partial}_{1}$ is the $\bar{\partial}$ operator with respect to $z^{\prime}$, and $\bar{\partial}_{2}$ is the $\bar{\partial}$-operator with respect to $z^{\prime \prime}$. Let $\mathcal{F}$ be the sheaf of germs of $C^{\infty}$ functions on $X$ which are holomorphic along the fibers $\left(\mathbb{C}^{*}\right)^{n-m}$, and let $\mathcal{F}^{r, s}$ be the sheaf of $(r, s)$-forms with respect to $\left\{d z_{1}, \ldots, d z_{m}, d \bar{z}_{1}, \ldots, d \bar{z}_{m}\right\}$ with coefficients in $\mathcal{F}$. Similarly, we denote by $\mathcal{F}(L)$ the sheaf of germs of $C^{\infty}$ functions with values in $L$ which are holomorphic along the fibers. Let $\mathcal{F}^{r, s}(L)$ be the sheaf of $(r, s)$-forms with respect to $\left\{d z_{1}, \ldots, d z_{m}, d \bar{z}_{1}, \ldots, d \bar{z}_{m}\right\}$ with coefficients in $\mathcal{F}(L)$.

The following proposition is due to Kazama and Umeno [6, Lemma 1.1].

Proposition 4.1. For any $r$, $s$ we have

$$
H^{p}\left(X, \mathcal{F}^{r, s}(L)\right)=0, \quad p \geq 1 .
$$

We have a resolution of $\mathcal{O}(L)$ :

$$
0 \longrightarrow \mathcal{O}(L) \longrightarrow \mathcal{F}(L) \stackrel{\bar{\partial}_{1}}{\longrightarrow} \mathcal{F}^{0,1}(L) \stackrel{\bar{\partial}_{1}}{\longrightarrow} \cdots \stackrel{\bar{\partial}_{1}}{\longrightarrow} \mathcal{F}^{0, m}(L) \longrightarrow 0 .
$$

By Proposition 4.1 we obtain the Dolbeault-Kazama isomorphisms

$$
H^{p}(X, \mathcal{O}(L)) \cong \frac{Z_{\bar{\partial}_{1}}\left(X, \mathcal{F}^{0, p}(L)\right)}{B_{\bar{\partial}_{1}}\left(X, \mathcal{F}^{0, p}(L)\right)}
$$

for $p \geq 1$, where we set

$$
Z_{\bar{\partial}_{1}}\left(X, \mathcal{F}^{0, p}(L)\right):=\left\{\varphi \in H^{0}\left(X, \mathcal{F}^{0, p}(L)\right) ; \bar{\partial}_{1} \varphi=0\right\}
$$

and $B_{\bar{\partial}_{1}}\left(X, \mathcal{F}^{0, p}(L)\right):=\bar{\partial}_{1} H^{0}\left(X, \mathcal{F}^{0, p-1}(L)\right)$.

\section{Fourier expansion}

We can identify $H^{0}(X, \mathcal{F})$ with the space of all $\Gamma$-periodic $C^{\infty}$ functions on $\mathbb{C}^{n}$ which are holomorphic with respect to $z^{\prime \prime}=\left(z_{m+1}, \ldots, z_{n}\right)$. Real coordinates $t=\left(t_{1}, \ldots, t_{2 n}\right)$ are written as $t=\left(t^{\prime}, t^{\prime \prime}\right)$, where $t^{\prime}=\left(t_{1}, \ldots, t_{n+m}\right)$ and $t^{\prime \prime}=\left(t_{n+m+1}, \ldots, t_{2 n}\right)$. Let $f \in$ $H^{0}(X, \mathcal{F})$. Since $\partial f / \partial \bar{z}_{m+j}=0$ for $j=1, \ldots, n-m$, we have the following Fourier expansion of $f$ :

$$
f(t)=\sum_{\sigma \in \mathbb{Z}^{n+m}} a^{\sigma} \exp \left(-2 \pi \sum_{i=m+1}^{n} \sigma_{i} t_{n+i}\right) \mathbf{e}\left(\left\langle\sigma, t^{\prime}\right\rangle\right),
$$

where $a^{\sigma}$ is a complex number and $\left\langle\sigma, t^{\prime}\right\rangle=\sum_{i=1}^{n+m} \sigma_{i} t_{i}$. The following lemma follows from the well known result for Fourier coefficients of $C^{\infty}$ functions.

LEMMA 5.1. Let $\left\{a^{\sigma} ; \sigma \in \mathbb{Z}^{n+m}\right\}$ be a sequence of complex numbers. We consider a formal series

$$
\sum_{\sigma \in \mathbb{Z}^{n+m}} a^{\sigma} \exp \left(-2 \pi \sum_{i=m+1}^{n} \sigma_{i} t_{n+i}\right) \mathbf{e}\left(\left\langle\sigma, t^{\prime}\right\rangle\right) .
$$


Then the series converges to a function in $H^{0}(X, \mathcal{F})$ if and only if for any $R>0$ and any $k>0$ we have

$$
\sup \left\{\left|a^{\sigma}\right| R^{\sum_{i=m+1}^{n}\left|\sigma_{i}\right|}|\sigma|^{k} ; \sigma \in \mathbb{Z}^{n+m}\right\}<\infty .
$$

We write $\sigma \in \mathbb{Z}^{n+m}$ as $\sigma=\left(\sigma^{\prime}, \sigma^{\prime \prime}, \sigma^{\prime \prime \prime}\right)$, where

$$
\sigma^{\prime}=\left(\sigma_{1}, \ldots, \sigma_{m}\right), \quad \sigma^{\prime \prime}=\left(\sigma_{m+1}, \ldots, \sigma_{n}\right) \quad \text { and } \quad \sigma^{\prime \prime \prime}=\left(\sigma_{n+1}, \ldots, \sigma_{n+m}\right) .
$$

If we set

$$
\begin{aligned}
f^{\sigma}(t) & :=a^{\sigma} \exp \left(-2 \pi \sum_{i=m+1}^{n} \sigma_{i} t_{n+i}\right) \mathbf{e}\left(\left\langle\sigma, t^{\prime}\right\rangle\right) \\
& =a^{\sigma} \mathbf{e}\left(\left\langle\sigma, t^{\prime}\right\rangle+\sqrt{-1}\left\langle\sigma^{\prime \prime}, t^{\prime \prime}\right\rangle\right)
\end{aligned}
$$

then (5.1) is rewritten as

$$
f(t)=\sum_{\sigma \in \mathbb{Z}^{n+m}} f^{\sigma}(t)
$$

By (2.2) we obtain

$$
\frac{\partial f^{\sigma}}{\partial \bar{z}_{j}}(t)=\pi \sum_{k=1}^{m} c_{k j} K_{\sigma, k} f^{\sigma}(t)
$$

for $j=1, \ldots, m$, where we set

$$
K_{\sigma, k}:=\sum_{\ell=1}^{n} \sigma_{\ell} s_{\ell k}-\sigma_{n+k}
$$

for $k=1, \ldots, m$. We note that

$$
K_{\sigma}:=\left(K_{\sigma, 1}, \ldots, K_{\sigma, m}\right)=\left(\sigma^{\prime}, \sigma^{\prime \prime}\right) S-\sigma^{\prime \prime \prime}
$$

for $\sigma=\left(\sigma^{\prime}, \sigma^{\prime \prime}, \sigma^{\prime \prime \prime}\right) \in \mathbb{Z}^{n+m}$. Put

$$
\widetilde{K}_{\sigma, j}:=\pi \sum_{\ell=1}^{m} c_{\ell j} K_{\sigma, \ell}
$$

for $j=1, \ldots, m$. We rewrite (5.3) as

$$
\frac{\partial f^{\sigma}}{\partial \bar{z}_{j}}(t)=\widetilde{K}_{\sigma, j} f^{\sigma}(t)
$$

for $j=1, \ldots, m$. Let $\widetilde{K}_{\sigma}:=\left(\widetilde{K}_{\sigma, 1}, \ldots, \widetilde{K}_{\sigma, m}\right)$. Then we have the relation

$$
\widetilde{K}_{\sigma}=\pi K_{\sigma}\left(c_{j k}\right)_{1 \leq j, k \leq m} \text {. }
$$

\section{Translation of $\bar{\partial}_{1}$-equations}

Let $L$ be a homogeneous line bundle over a toroidal group $X=\mathbb{C}^{n} / \Gamma$ with rank $\Gamma=n+m$. It is given by a representation $\rho=\mathbf{e}(d)$ of $\Gamma$, where $d: \Gamma \longrightarrow \mathbb{R}$ is a homomorphism having the properties stated in Lemma 3.3. We define a linear polynomial $a(t)$ in $t$ by

$$
a(t):=-\sum_{i=1}^{m} d\left(e_{i}\right) t_{i}-\sum_{i=1}^{m} d\left(s_{i}\right) t_{n+i} .
$$


It is easy to check that

$$
a(t+\gamma)+d(\gamma)-a(t)=0
$$

for any $\gamma \in \Gamma$ and any $t \in \mathbb{R}^{2 n}$. By (2.2) we obtain $\partial a / \partial \bar{z}_{i}=0$ for $i=m+1, \ldots, n$. Then we have $a \in H^{0}\left(\mathbb{C}^{n}, \pi^{*} \mathcal{F}\right)$, where $\pi: \mathbb{C}^{n} \longrightarrow X$ is the projection. We set

$$
F(t):=\mathbf{e}(a(t)) .
$$

We can consider $H^{0}\left(X, \mathcal{F}^{r, s}\right), H^{0}\left(X, \mathcal{F}^{r, s}(L)\right)$ and $H^{p}(X, \mathcal{O}(L))$ as Fréchet spaces in the usual manner. Then the multiplicity by $F(t)$ gives isomorphisms between Fréchet spaces

$$
F: H^{0}\left(X, \mathcal{F}^{r, s}(L)\right) \longrightarrow H^{0}\left(X, \mathcal{F}^{r, s}\right), \quad \varphi \longmapsto F \varphi
$$

by (6.2). We set

$$
G(t):=F(t)^{-1}=\mathbf{e}(-a(t)) .
$$

Then, for any $\varphi \in H^{0}\left(X, \mathcal{F}^{0, p}(L)\right)$ there exists uniquely $\phi \in H^{0}\left(X, \mathcal{F}^{0, p}\right)$ such that

$$
\varphi=G \phi
$$

By a straight calculation using (2.1) we obtain

$$
\begin{aligned}
-a(t)= & \frac{1}{2} \sum_{j=1}^{m}\left(d\left(e_{j}\right)-\sqrt{-1} \sum_{i=1}^{m}\left(d\left(s_{i}\right) c_{i j}-d\left(e_{i}\right) \sum_{k=1}^{m} a_{i k} c_{k j}\right)\right) z_{j} \\
& +\frac{1}{2} \sum_{j=1}^{m}\left(d\left(e_{j}\right)+\sqrt{-1} \sum_{i=1}^{m}\left(d\left(s_{i}\right) c_{i j}-d\left(e_{i}\right) \sum_{k=1}^{m} a_{i k} c_{k j}\right)\right) \bar{z}_{j} .
\end{aligned}
$$

Then, setting

$$
\alpha_{j}:=d\left(e_{j}\right)-\sqrt{-1} \sum_{i=1}^{m}\left(d\left(s_{i}\right) c_{i j}-d\left(e_{i}\right) \sum_{k=1}^{m} a_{i k} c_{k j}\right)
$$

we have

$$
-a(t)=\frac{1}{2} \sum_{j=1}^{m}\left(\alpha_{j} z_{j}+\bar{\alpha}_{j} \bar{z}_{j}\right)
$$

We denote

$$
A_{1}:=\left(a_{i k}\right)_{1 \leq i, k \leq m} \quad \text { and } \quad C_{1}:=\left(c_{k j}\right)_{1 \leq k, j \leq m} .
$$

Then we have

$$
\begin{aligned}
\left(\alpha_{1}, \ldots, \alpha_{m}\right)= & \left(d\left(e_{1}\right), \ldots, d\left(e_{m}\right)\right) \\
& -\sqrt{-1}\left\{\left(d\left(s_{1}\right), \ldots, d\left(s_{m}\right)\right)-\left(d\left(e_{1}\right), \ldots, d\left(e_{m}\right)\right) A_{1}\right\} C_{1} .
\end{aligned}
$$

Operating $\bar{\partial}_{1}$ on $G(t)$, we obtain

$$
\bar{\partial}_{1} G=\sum_{j=1}^{m} \frac{\partial G}{\partial \bar{z}_{j}} d \bar{z}_{j}=\pi \sqrt{-1} G \sum_{j=1}^{m} \bar{\alpha}_{j} d \bar{z}_{j} .
$$

We set $\Phi_{0}:=\sum_{j=1}^{m} \beta_{j} d \bar{z}_{j}$, where $\beta_{j}:=\pi \sqrt{-1} \bar{\alpha}_{j}$. Then we have

$$
\bar{\partial}_{1} G=G \Phi_{0} .
$$


By relation (6.3) we obtain

$$
\bar{\partial}_{1} \varphi=G\left(\Phi_{0} \wedge \phi+\bar{\partial}_{1} \phi\right)
$$

Therefore, $\bar{\partial}_{1} \varphi=0$ if and only if

$$
\Phi_{0} \wedge \phi+\bar{\partial}_{1} \phi=0
$$

We assume that there exists $\eta \in H^{0}\left(X, \mathcal{F}^{0, p-1}(L)\right)$ with $\bar{\partial}_{1} \eta=\varphi$. Then we can take $\psi \in H^{0}\left(X, \mathcal{F}^{0, p-1}\right)$ such that $\eta=G \psi$ and

$$
\phi=\Phi_{0} \wedge \psi+\bar{\partial}_{1} \psi
$$

by (6.6). Therefore the problem of finding $\eta$ with $\bar{\partial}_{1} \eta=\varphi$ for $\varphi \in Z_{\bar{\partial}_{1}}\left(X, \mathcal{F}^{0, p}(L)\right)$ is translated to the following problem.

Problem. For any $\phi \in H^{0}\left(X, \mathcal{F}^{0, p}\right)$ satisfying (6.7), does there exist $\psi \in H^{0}\left(X, \mathcal{F}^{0, p-1}\right)$ such that the equation (6.8) holds?

\section{Formal solutions}

We may assume that the homogeneous line bundle $L$ is not analytically trivial. Then we have $a(t) \neq 0$. This means $\left(\bar{\alpha}_{1}, \ldots, \bar{\alpha}_{m}\right) \neq(0, \ldots, 0)$. Hence we have

$$
\left(\beta_{1}, \ldots, \beta_{m}\right)=\pi \sqrt{-1}\left(\bar{\alpha}_{1}, \ldots, \bar{\alpha}_{m}\right) \neq(0, \ldots, 0) .
$$

Consider equations

$$
\widetilde{K}_{\sigma, j}+\beta_{j}=0, \quad j=1, \ldots, m .
$$

It is easily seen that (7.1) is equivalent to

$$
K_{\sigma} C_{1}+\sqrt{-1}\left(\bar{\alpha}_{1}, \ldots, \bar{\alpha}_{m}\right)=(0, \ldots, 0) .
$$

Furthermore, (7.2) is equivalent to

$$
\left\{\begin{array}{l}
\left(\sigma^{\prime}, \sigma^{\prime \prime}\right) \operatorname{Re}(S)+\sigma^{\prime \prime \prime}+\left(\operatorname{Im}\left(\alpha_{1}\right), \ldots, \operatorname{Im}\left(\alpha_{m}\right)\right) C_{1}^{-1}=0 \\
\left(\sigma^{\prime}, \sigma^{\prime \prime}\right) \operatorname{Im}(S)+\left(\operatorname{Re}\left(\alpha_{1}\right), \ldots, \operatorname{Re}\left(\alpha_{m}\right)\right) C_{1}^{-1}=0
\end{array}\right.
$$

by (5.5). We note that (7.3) does not hold for $\sigma=0$.

LEMMA 7.1. If there exists $\sigma_{0}=\left(\sigma_{0}^{\prime}, \sigma_{0}^{\prime \prime}, \sigma_{0}^{\prime \prime \prime}\right) \in \mathbb{Z}^{n+m} \backslash\{0\}$ satisfying (7.3), then it is unique.

Proof. Suppose that $\sigma=\left(\sigma^{\prime}, \sigma^{\prime \prime}, \sigma^{\prime \prime \prime}\right) \in \mathbb{Z}^{n+m} \backslash\{0\}$ also satisfies (7.3). Then we have

$$
\left(\sigma^{\prime}-\sigma_{0}^{\prime}, \sigma^{\prime \prime}-\sigma_{0}^{\prime \prime}\right) S+\sigma^{\prime \prime \prime}-\sigma_{0}^{\prime \prime \prime}=0 .
$$

By the irrationality condition (IS) in Section 2 we obtain $\sigma-\sigma_{0}=0$.

We define

$$
Z:= \begin{cases}\mathbb{Z}^{n+m} \backslash\left\{\sigma_{0}\right\} & \text { if there exists } \sigma_{0} \text { with (7.3) } \\ \mathbb{Z}^{n+m} & \text { otherwise. }\end{cases}
$$


For any $\sigma \in \mathbb{Z}^{n+m}$ we denote by $j(\sigma)$ the smallest integer $j$ satisfying

$$
\left|\widetilde{K}_{\sigma, j}+\beta_{j}\right|=\max \left\{\left|\widetilde{K}_{\sigma, k}+\beta_{k}\right| ; k=1, \ldots, m\right\} .
$$

We note that $j\left(\sigma_{0}\right)=1$ for $\sigma_{0}$ satisfying (7.3).

Every $\phi \in H^{0}\left(X, \mathcal{F}^{0, p}\right)$ has the Fourier expansion

$$
\phi=\sum_{\sigma \in \mathbb{Z}^{n+m}} \phi^{\sigma}, \quad \phi^{\sigma}=\mathbf{e}\left(\left\langle\sigma, t^{\prime}\right\rangle+\sqrt{-1}\left(\left\langle\sigma^{\prime \prime}, t^{\prime \prime}\right\rangle\right) \phi_{c}^{\sigma},\right.
$$

where $\phi_{c}^{\sigma}$ is a $(0, p)$-form with constant coefficients. For any $\sigma \in \mathbb{Z}^{n+m}$ we have the unique representation

$$
\begin{aligned}
\phi^{\sigma}= & \sum_{\substack{1 \leq i_{1}<\cdots<i_{p-1} \leq m\\
}}\left(\phi_{j(\sigma) i_{1} \ldots i_{p-1}}^{\sigma} d \bar{z}_{j(\sigma)}+\sum_{j \notin\left\{j(\sigma), i_{1}, \ldots, i_{p-1}\right\}} \phi_{j i_{1} \ldots i_{p-1}}^{\sigma} d \bar{z}_{j}\right) \\
& \wedge d \bar{z}_{i_{1}} \wedge \cdots \wedge d \bar{z}_{i_{p-1}},
\end{aligned}
$$

where

$$
\phi_{k i_{1} \ldots i_{p-1}}^{\sigma}=a_{k i_{1} \ldots i_{p-1}}^{\sigma} \mathbf{e}\left(\left\langle\sigma, t^{\prime}\right\rangle+\sqrt{-1}\left\langle\sigma^{\prime \prime}, t^{\prime \prime}\right\rangle\right), \quad a_{k i_{1} \ldots i_{p-1}}^{\sigma} \in \mathbb{C} .
$$

Here we mean that $\phi_{j(\sigma) i_{1} \ldots i_{p-1}}^{\sigma}=0$ if $j(\sigma) \in\left\{i_{1}, \ldots, i_{p-1}\right\}$. Since

$$
\begin{aligned}
\bar{\partial}_{1} \phi^{\sigma}= & \sum_{1 \leq i_{1}<\cdots<i_{p-1} \leq m}\left\{\sum_{j \notin\left\{j(\sigma), i_{1}, \ldots, i_{p-1}\right\}}\left(\frac{\partial \phi_{j i_{1} \ldots i_{p-1}}^{\sigma}}{\partial \bar{z}_{j(\sigma)}}-\frac{\partial \phi_{j(\sigma) i_{1} \ldots i_{p-1}}^{\sigma}}{\partial \bar{z}_{j}}\right) d \bar{z}_{j(\sigma)} \wedge d \bar{z}_{j}\right\} \\
& \wedge d \bar{z}_{i_{1}} \wedge \cdots \wedge d \bar{z}_{i_{p-1}},
\end{aligned}
$$

we have

$$
\begin{aligned}
\Phi_{0} \wedge \phi^{\sigma}+\bar{\partial}_{1} \phi^{\sigma}= & \sum_{\substack{1 \leq i_{1}<\cdots<i_{p-1} \leq m \\
j \notin\left\{j(\sigma), i_{1}, \ldots, i_{p-1}\right\}}}\left(\frac{\partial \phi_{j i_{1} \ldots i_{p-1}}^{\sigma}}{\partial \bar{z}_{j(\sigma)}}\right. \\
& \left.-\frac{\partial \phi_{j(\sigma) i_{1} \ldots i_{p-1}}^{\sigma}}{\partial \bar{z}_{j}}+\beta_{j(\sigma)} \phi_{j i_{1} \ldots i_{p-1}}^{\sigma}-\beta_{j} \phi_{j(\sigma) i_{1} \ldots i_{p-1}}^{\sigma}\right) \\
& \left.\times d \bar{z}_{j(\sigma)} \wedge d \bar{z}_{j}+\sum_{\substack{j, k \notin\left\{j(\sigma), i_{1}, \ldots, i_{p-1}\right\} \\
j \neq k}} \beta_{k} \phi_{j i_{1} \ldots i_{p-1}}^{\sigma} d \bar{z}_{k} \wedge d \bar{z}_{j}\right\} \\
& \wedge d \bar{z}_{i_{1}} \wedge \cdots \wedge d \bar{z}_{i_{p-1} .} .
\end{aligned}
$$

It follows from (5.6) that

$$
\frac{\partial \phi_{j i_{1} \ldots i_{p-1}}^{\sigma}}{\partial \bar{z}_{k}}=\widetilde{K}_{\sigma, k} \phi_{j i_{1} \ldots i_{p-1}}^{\sigma} .
$$

If $\phi$ satisfies (6.7), then

$$
\left(\widetilde{K}_{\sigma, j(\sigma)}+\beta_{j(\sigma)}\right) \phi_{j i_{1} \ldots i_{p-1}}^{\sigma}=\left(\widetilde{K}_{\sigma, j}+\beta_{j}\right) \phi_{j(\sigma) i_{1} \ldots i_{p-1}}^{\sigma}
$$

for $j \notin\left\{j(\sigma), i_{1}, \ldots, i_{p-1}\right\}$ and

$$
\beta_{k} \phi_{j i_{1} \ldots i_{p-1}}^{\sigma}=0
$$

for $j, k \notin\left\{j(\sigma), i_{1}, \ldots, i_{p-1}\right\}$ with $j \neq k$. 
Assume that there exists $\psi \in H^{0}\left(X, \mathcal{F}^{0, p-1}\right)$ satisfying (6.8). We have the expansion $\psi=\sum_{\sigma \in \mathbb{Z}^{n+m}} \psi^{\sigma}$ as (7.4). Each $\psi^{\sigma}$ has the representation

$$
\psi^{\sigma}=\sum_{1 \leq i_{1}<\cdots<i_{p-1} \leq m} \psi_{i_{1} \ldots i_{p-1}}^{\sigma} d \bar{z}_{i_{1}} \wedge \cdots \wedge d \bar{z}_{i_{p-1}},
$$

where

$$
\psi_{i_{1} \ldots i_{p-1}}^{\sigma}=b_{i_{1} \ldots i_{p-1}}^{\sigma} \mathbf{e}\left(\left\langle\sigma, t^{\prime}\right\rangle+\sqrt{-1}\left\langle\sigma^{\prime \prime}, t^{\prime \prime}\right\rangle\right), \quad b_{i_{1} \ldots i_{p-1}}^{\sigma} \in \mathbb{C} .
$$

Since

$$
\begin{aligned}
\bar{\partial}_{1} \psi^{\sigma}= & \sum_{1 \leq i_{1}<\cdots<i_{p-1} \leq m}\left(\frac{\partial \psi_{i_{1} \ldots i_{p-1}}^{\sigma}}{\partial \bar{z}_{j(\sigma)}} d \bar{z}_{j(\sigma)}+\sum_{j \notin\left\{j(\sigma), i_{1}, \ldots, i_{p-1}\right\}} \frac{\partial \psi_{i_{1} \ldots i_{p-1}}^{\sigma}}{\partial \bar{z}_{j}} d \bar{z}_{j}\right) \\
& \wedge d \bar{z}_{i_{1}} \wedge \cdots \wedge d \bar{z}_{i_{p-1}}
\end{aligned}
$$

and

$$
\Phi_{0} \wedge \psi^{\sigma}=\sum_{1 \leq i_{1}<\cdots<i_{p-1} \leq m}\left(\sum_{k=1}^{m} \beta_{k} \psi_{i_{1} \ldots i_{p-1}}^{\sigma} d \bar{z}_{k}\right) \wedge d \bar{z}_{i_{1}} \wedge \cdots \wedge d \bar{z}_{i_{p-1}},
$$

we have

$$
\frac{\partial \psi_{i_{1} \ldots i_{p-1}}^{\sigma}}{\partial \bar{z}_{j(\sigma)}}+\beta_{j(\sigma)} \psi_{i_{1} \ldots i_{p-1}}^{\sigma}=\phi_{j(\sigma) i_{1} \ldots i_{p-1}}^{\sigma}
$$

and

$$
\frac{\partial \psi_{i_{1} \ldots i_{p-1}}^{\sigma}}{\partial \bar{z}_{j}}+\beta_{j} \psi_{i_{1} \ldots i_{p-1}}^{\sigma}=\phi_{j i_{1} \ldots i_{p-1}}^{\sigma}
$$

for $j \notin\left\{j(\sigma), i_{1}, \ldots, i_{p-1}\right\}$. It follows from (5.6) that (7.10) and (7.11) are equivalent to

$$
\left(\widetilde{K}_{\sigma, j(\sigma)}+\beta_{j(\sigma)}\right) \psi_{i_{1} \ldots i_{p-1}}^{\sigma}=\phi_{j(\sigma) i_{1} \ldots i_{p-1}}^{\sigma}
$$

and

$$
\left(\widetilde{K}_{\sigma, j}+\beta_{j}\right) \psi_{i_{1} \ldots i_{p-1}}^{\sigma}=\phi_{j i_{1} \ldots i_{p-1}}^{\sigma}
$$

for $j \notin\left\{j(\sigma), i_{1}, \ldots, i_{p-1}\right\}$, respectively. Then for any $\sigma \in Z$ and any $i_{1}, \ldots, i_{p-1}$ with $1 \leq i_{1}<\cdots<i_{p-1} \leq m$ we set

$$
\psi_{i_{1} \ldots i_{p-1}}^{\sigma}:=\frac{\phi_{j(\sigma) i_{1} \ldots i_{p-1}}^{\sigma}}{\widetilde{K}_{\sigma, j(\sigma)}+\beta_{j(\sigma)}} .
$$

We note that $\left\{\psi_{i_{1} \ldots i_{p-1}}^{\sigma}\right\}$ satisfy (7.13) for $\left\{\phi_{j i_{1} \ldots i_{p-1}}^{\sigma}\right\}$ with the property (7.7). We define $\psi^{\sigma}$ by (7.9). We consider the formal sum

$$
\psi:=\sum_{\sigma \in Z} \psi^{\sigma}
$$

Then, if $Z=\mathbb{Z}^{n+m}$, we have

$$
\phi=\Phi_{0} \wedge \psi+\bar{\partial}_{1} \psi
$$

and if $Z=\mathbb{Z}^{n+m} \backslash\left\{\sigma_{0}\right\}$, we have

$$
\phi=\Phi_{0} \wedge \psi+\bar{\partial}_{1} \psi+\phi^{\sigma_{0}},
$$

where

$$
\phi^{\sigma_{0}}=\mathbf{e}\left(\left\langle\sigma_{0}, t^{\prime}\right\rangle+\sqrt{-1}\left\langle\sigma_{0}^{\prime \prime}, t^{\prime \prime}\right\rangle\right) \phi_{c}^{\sigma_{0}} .
$$




\section{Conditions}

We define $d(L) \in \mathbb{C}^{m}$ for any homogeneous line bundle $L$ over a toroidal group $X$ by

$$
d(L):=\sqrt{-1}\left(\bar{\alpha}_{1}, \ldots, \bar{\alpha}_{m}\right) C_{1}^{-1} .
$$

Then we have

$$
\left(\widetilde{K}_{\sigma, 1}+\beta_{1}, \ldots, \widetilde{K}_{\sigma, m}+\beta_{m}\right)=\pi\left(K_{\sigma}+d(L)\right) C_{1} .
$$

Noting $K_{\sigma}+d(L)=-\sigma^{\prime \prime \prime}+d(L) \neq 0$ for any $\sigma \in Z$ with $\left(\sigma^{\prime}, \sigma^{\prime \prime}\right)=(0,0)$, we set

$$
m_{0}:=\min \left\{\left\|-\sigma^{\prime \prime \prime}+d(L)\right\| ;\left(0,0, \sigma^{\prime \prime \prime}\right) \in Z\right\} .
$$

Then we have

$$
m_{0} \leq\left\|K_{\sigma}+d(L)\right\|
$$

for any $\sigma=\left(0,0, \sigma^{\prime \prime \prime}\right) \in Z$. We consider the following condition $(H)_{S}$.

There exists $r>0$ such that

$$
\left\|K_{\sigma}+d(L)\right\| \geq r^{-\left|\left(\sigma^{\prime}, \sigma^{\prime \prime}\right)\right|}
$$

for all $\sigma \in Z$ with $\left(\sigma^{\prime}, \sigma^{\prime \prime}\right) \neq(0,0)$.

LEMma 8.1. The condition $(H)_{S}$ is equivalent to the following condition $(H)_{S}^{\prime}$.

There exist constants $C>0$ and $a \geq 0$ such that

$$
\left\|K_{\sigma}+d(L)\right\| \geq C \exp \left(-a\left|\left(\sigma^{\prime}, \sigma^{\prime \prime}\right)\right|\right)
$$

for all $\sigma \in Z$ with $\left(\sigma^{\prime}, \sigma^{\prime \prime}\right) \neq(0,0)$.

Proof. Let $r>0$ be a constant for which the condition $(H)_{S}$ is satisfied. For any $r^{\prime}$ with $r^{\prime} \geq r$ the condition $(H)_{S}$ holds. Therefore, we may assume $r \geq 1$. Then the condition $(H)_{S}^{\prime}$ holds for $C=1$ and $a:=\log r$.

Conversely, we suppose that the condition $(H)_{S}^{\prime}$ is satisfied for some constants $C>0$ and $a \geq 0$. We take $r>0$ with

$$
\log r>\max \{a-\log C, a\} .
$$

Then we have

$$
r^{-\lambda}<C e^{-a \lambda} \quad(\lambda \geq 1)
$$

Therefore we obtain

$$
r^{-\left|\left(\sigma^{\prime}, \sigma^{\prime \prime}\right)\right|}<C \exp \left(-a\left|\left(\sigma^{\prime}, \sigma^{\prime \prime}\right)\right|\right) \leq\left\|K_{\sigma}+d(L)\right\|
$$

for any $\sigma \in Z$ with $\left(\sigma^{\prime}, \sigma^{\prime \prime}\right) \neq(0,0)$.

LEMMA 8.2. The condition $(H)_{S}^{\prime}$ is equivalent to the following condition $(H)_{S}^{\prime \prime}$.

There exist constants $C>0$ and $a \geq 0$ such that

$$
\left\|K_{\sigma}+d(L)\right\| \geq C \exp \left(-a\left|\sigma^{\prime \prime}\right|\right)
$$

for all $\sigma \in Z$ with $\left(\sigma^{\prime}, \sigma^{\prime \prime}\right) \neq(0,0)$. 
Proof. Since

$$
\exp \left(-a\left|\left(\sigma^{\prime}, \sigma^{\prime \prime}\right)\right|\right) \leq \exp \left(-a\left|\sigma^{\prime \prime}\right|\right)
$$

the condition $(H)_{S}^{\prime \prime}$ obviously implies the condition $(H)_{S}^{\prime}$.

We suppose that the condition $(H)_{S}^{\prime}$ is satisfied. It is trivial that we can take constants $C>0$ and $a \geq 0$ satisfying the inequality in $(H)_{S}^{\prime \prime}$ for any $\sigma \in Z$ with $\left(\sigma^{\prime}, \sigma^{\prime \prime}\right) \neq(0,0)$ and $\left\|K_{\sigma}+d(L)\right\|>1$. Therefore, it suffices to consider the condition $(H)_{S}^{\prime \prime}$ for the set

$$
\Sigma:=\left\{\sigma \in Z ;\left(\sigma^{\prime}, \sigma^{\prime \prime}\right) \neq(0,0),\left\|K_{\sigma}+d(L)\right\| \leq 1\right\} .
$$

We write $d(L)=\left(d(L)_{1}, \ldots, d(L)_{m}\right) \in \mathbb{C}^{m}$. Let $\sigma \in \Sigma$. For any $\ell=1, \ldots, m$ we have

$$
\begin{aligned}
\left|\sum_{j=1}^{m} \sigma_{j} \operatorname{Im}\left(s_{j \ell}\right)\right| & \leq\left|\sum_{j=1}^{m} \sigma_{j} s_{j \ell}-\sigma_{n+\ell}\right| \\
& \leq\left|\sum_{j=1}^{n} \sigma_{j} s_{j \ell}-\sigma_{n+\ell}+d(L)_{\ell}\right|+\left|d(L)_{\ell}\right|+\left|\sum_{j=m+1}^{n} \sigma_{j} s_{j \ell}\right| \\
& \leq\left\|K_{\sigma}+d(L)\right\|+\|d(L)\|+\sum_{j=m+1}^{n}\left|s_{j \ell} \| \sigma_{j}\right| \\
& \leq 1+\|d(L)\|+\gamma_{1}\left|\sigma^{\prime \prime}\right|
\end{aligned}
$$

where we set

$$
\gamma_{1}:=\max \left\{\left|s_{j \ell}\right| ; 1 \leq j \leq n, 1 \leq \ell \leq m\right\} .
$$

Since $\operatorname{det}\left(\operatorname{Im}\left(S_{1}\right)\right) \neq 0$, there exists $\gamma_{2}>0$ such that

$$
\max \left\{\left|\sum_{j=1}^{m} \sigma_{j} \operatorname{Im}\left(s_{j \ell}\right)\right| ; \ell=1, \ldots, m\right\} \geq \gamma_{2} \sum_{j=1}^{m}\left|\sigma_{j}\right| .
$$

By (8.3) and (8.4) we can take constants $\gamma_{1}^{\prime}>0$ and $\gamma_{2}^{\prime}>0$ such that

$$
\sum_{j=1}^{m}\left|\sigma_{j}\right| \leq \gamma_{1}^{\prime}+\gamma_{2}^{\prime} \sum_{j=m+1}^{n}\left|\sigma_{j}\right| \text {. }
$$

Let $C>0$ and $a \geq 0$ be constants satisfying the condition $(H)_{S}^{\prime}$. Then we obtain

$$
\begin{aligned}
\left\|K_{\sigma}+d(L)\right\| & \geq C \exp \left(-a\left|\left(\sigma^{\prime}, \sigma^{\prime \prime}\right)\right|\right) \\
& =C \exp \left(-a \sum_{i=1}^{m}\left|\sigma_{i}\right|\right) \exp \left(-a \sum_{j=m+1}^{n}\left|\sigma_{j}\right|\right) \\
& \geq C \exp \left(-a \gamma_{1}^{\prime}\right) \exp \left(-a\left(1+\gamma_{2}^{\prime}\right) \sum_{j=m+1}^{n}\left|\sigma_{j}\right|\right)
\end{aligned}
$$

for $\sigma \in \Sigma$. This finishes the proof. 


\section{Cohomology groups}

The following theorem is our main result.

THEOREM 9.1. Let $L \longrightarrow X$ be a homogeneous line bundle over a toroidal group $X=$ $\mathbb{C}^{n} / \Gamma$ with rank $\Gamma=n+m$. We assume that $L$ is not analytically trivial. We consider the condition $(H)_{S}$ in Section 8 and the set $Z$ defined after Lemma 7.1. Then one of the following cases holds.

(I) The case that the condition $(H)_{S}$ is satisfied for $d(L)$ :

(i) if $Z=\mathbb{Z}^{n+m}$, then $H^{p}(X, \mathcal{O}(L))=0$ for $p \geq 1$;

(ii) if $Z=\mathbb{Z}^{n+m} \backslash\left\{\sigma_{0}\right\}$, then

$$
H^{p}(X, \mathcal{O}(L)) \cong H^{p}(\mathbb{T}, \mathcal{O}) \text { for } p \geq 1,
$$

where $\mathbb{T}$ is an m-dimensional complex torus over which $X$ is a principal $\left(\mathbb{C}^{*}\right)^{n-m}$-bundle.

(II) The case that the condition $(H)_{S}$ is not satisfied for $d(L)$.

For any $p$ with $1 \leq p \leq m, H^{p}(X, \mathcal{O}(L))$ is a non-Hausdorff Fréchet space. Then it is of infinite dimension.

When $X$ is a complex torus, the case (i) in (I) holds.

We need the following lemma for the proof of Theorem 9.1.

LEMMA 9.2. Under the assumption of Theorem 9.1, $B_{\bar{\partial}_{1}}\left(X, \mathcal{F}^{0, p}(L)\right)$ is a closed subspace of $Z_{\bar{\partial}_{1}}\left(X, \mathcal{F}^{0, p}(L)\right)$ if and only if the space

$$
\left\{\Phi_{0} \wedge \psi+\bar{\partial}_{1} \psi ; \psi \in H^{0}\left(X, \mathcal{F}^{0, p-1}\right)\right\}
$$

is a closed subspace of

$$
\left\{\phi \in H^{0}\left(X, \mathcal{F}^{0, p}\right) ; \Phi_{0} \wedge \phi+\bar{\partial}_{1} \phi=0\right\} .
$$

Proof. For any sequence $\left\{\varphi^{(k)}\right\}$ in $H^{0}\left(X, \mathcal{F}^{0, p-1}(L)\right)$ there exists uniquely a sequence $\left\{\phi^{(k)}\right\}$ in $H^{0}\left(X, \mathcal{F}^{0, p-1}\right)$ such that $\varphi^{(k)}=G \phi^{(k)}$ and

$$
\bar{\partial}_{1} \varphi^{(k)}=G\left(\Phi_{0} \wedge \phi^{(k)}+\bar{\partial}_{1} \phi^{(k)}\right) .
$$

Then $\left\{\bar{\partial}_{1} \varphi^{(k)}\right\}$ converges to some $\eta \in Z_{\bar{\partial}_{1}}\left(X, \mathcal{F}^{0, p}(L)\right)$ if and only if $\left\{\Phi_{0} \wedge \phi^{(k)}+\bar{\partial}_{1} \phi^{(k)}\right\}$ converges to $\tau$ with $\eta=G \tau$. Therefore, there exists $\varphi \in H^{0}\left(X, \mathcal{F}^{0, p-1}(L)\right)$ with $\eta=\bar{\partial}_{1} \varphi$ if and only if there exists $\phi \in H^{0}\left(X, \mathcal{F}^{0, p-1}\right)$ such that

$$
\tau=\Phi_{0} \wedge \phi+\bar{\partial}_{1} \phi
$$

Proof of Theorem 9.1. We first show that the condition $(H)_{S}$ is satisfied and $Z=\mathbb{Z}^{2 n}$ when $X$ is a complex torus. In this case, the matrix $S$ in the period matrix $P$ is a complex $(n, n)$-matrix with $\operatorname{det}(\operatorname{Im}(S)) \neq 0$. We may assume that the homomorphism $d: \Gamma \longrightarrow \mathbb{R}$ corresponding to $L$ satisfies $0 \leq d\left(e_{i}\right), d\left(s_{i}\right)<1$ for all $i=1, \ldots, n$. Since $L$ is not analytically trivial, there exists $i$ such that $d\left(e_{i}\right)$ or $d\left(s_{i}\right)$ is not zero. Any $\sigma \in \mathbb{Z}^{2 n}$ is written as $\sigma=\left(\sigma^{\prime}, \sigma^{\prime \prime \prime}\right)$, $\sigma^{\prime}, \sigma^{\prime \prime \prime} \in \mathbb{Z}^{n}$. We have $K_{\sigma}=\sigma^{\prime} S-\sigma^{\prime \prime \prime}$ for $\sigma=\left(\sigma^{\prime}, \sigma^{\prime \prime \prime}\right)$. Noting $A_{1}=A=\operatorname{Re}(S)$ and 
$C_{1}=C=(\operatorname{Im}(S))^{-1}$, we obtain

$$
\begin{aligned}
d(L)= & -\left\{\left(d\left(s_{1}\right), \ldots, d\left(s_{n}\right)\right)-\left(d\left(e_{1}\right), \ldots, d\left(e_{n}\right)\right) \operatorname{Re}(S)\right\} \\
& +\sqrt{-1}\left(d\left(e_{1}\right), \ldots, d\left(e_{n}\right)\right) \operatorname{Im}(S) .
\end{aligned}
$$

Then we have

$$
\begin{aligned}
& \operatorname{Re}\left(K_{\sigma}+d(L)\right)=\left\{\sigma^{\prime}+\left(d\left(e_{1}\right), \ldots, d\left(e_{n}\right)\right)\right\} \operatorname{Re}(S)-\left\{\sigma^{\prime \prime \prime}+\left(d\left(s_{1}\right), \ldots, d\left(s_{n}\right)\right)\right\}, \\
& \operatorname{Im}\left(K_{\sigma}+d(L)\right)=\left\{\sigma^{\prime}+\left(d\left(e_{1}\right), \ldots, d\left(e_{n}\right)\right)\right\} \operatorname{Im}(S) .
\end{aligned}
$$

If $\left(d\left(e_{1}\right), \ldots, d\left(e_{n}\right)\right) \neq(0, \ldots, 0)$, then there exists a constant $\delta_{1}>0$ such that

$$
\left\|\operatorname{Im}\left(K_{\sigma}+d(L)\right)\right\|>\delta_{1}
$$

for all $\sigma \in \mathbb{Z}^{2 n}$. If $\left(d\left(e_{1}\right), \ldots, d\left(e_{n}\right)\right)=(0, \ldots, 0)$, then $\left(d\left(s_{1}\right), \ldots, d\left(s_{n}\right)\right) \neq(0, \ldots, 0)$. In this case $\left\|\operatorname{Im}\left(K_{\sigma}+d(L)\right)\right\|=0$ holds only when $\sigma^{\prime}=0$. There exists a constant $\delta_{2}>0$ such that

$$
\left\|\sigma^{\prime \prime \prime}+\left(d\left(s_{1}\right), \ldots, d\left(s_{n}\right)\right)\right\|>\delta_{2}
$$

for all $\sigma^{\prime \prime \prime} \in \mathbb{Z}^{n}$. On the other hand, we have a constant $\delta_{1}^{\prime}>0$ such that

$$
\left\|\left\{\sigma^{\prime}+\left(d\left(e_{1}\right), \ldots, d\left(e_{n}\right)\right)\right\} \operatorname{Im}(S)\right\|>\delta_{1}^{\prime}
$$

for any $\sigma^{\prime} \in \mathbb{Z}^{n} \backslash\{0\}$. Therefore, we can take a constant $\delta>0$ such that

$$
\left\|K_{\sigma}+d(L)\right\|>\delta
$$

for all $\sigma \in \mathbb{Z}^{2 n}$. This shows that the condition $(H)_{S}$ is satisfied and $Z=\mathbb{Z}^{2 n}$.

Next we consider the general toroidal groups. Take any $\phi \in H^{0}\left(X, \mathcal{F}^{0, p}\right)$ satisfying (6.7). Then we have the formal solution $\psi=\sum_{\sigma \in Z} \psi^{\sigma}$ of (7.16) or (7.17), where $\psi^{\sigma}$ has the representation (7.9).

(I) We assume that the condition $(H)_{S}$ is satisfied. By the definition of $j(\sigma)$ we have

$$
\left\|\widetilde{K}_{\sigma}+\left(\beta_{1}, \ldots, \beta_{m}\right)\right\| \leq \sqrt{m}\left|\widetilde{K}_{\sigma, j(\sigma)}+\beta_{j(\sigma)}\right| .
$$

There exists a positive constant $M$ such that

$$
\left\|K_{\sigma}+d(L)\right\| \leq M\left\|\widetilde{K}_{\sigma}+\left(\beta_{1}, \ldots, \beta_{m}\right)\right\|
$$

by (8.1). Then we obtain

$$
\left|\widetilde{K}_{\sigma, j(\sigma)}+\beta_{j(\sigma)}\right| \geq \frac{1}{\sqrt{m} M}\left\|K_{\sigma}+d(L)\right\| .
$$

By Lemmas 8.1 and 8.2 we can take $C>0$ and $a \geq 0$ such that

$$
\left\|K_{\sigma}+d(L)\right\| \geq C \exp \left(-a\left|\sigma^{\prime \prime}\right|\right)
$$

for any $\sigma \in Z$ with $\left(\sigma^{\prime}, \sigma^{\prime \prime}\right) \neq(0,0)$. Since $\psi_{i_{1} \ldots i_{p-1}}^{\sigma}$ is defined by (7.14), it follows from (9.1) and (9.2) that

$$
\left|\psi_{i_{1} \ldots i_{p-1}}^{\sigma}\right| \leq \frac{\sqrt{m} M}{C} \exp \left(a\left|\sigma^{\prime \prime}\right|\right)\left|\phi_{j(\sigma) i_{1} \ldots i_{p-1}}^{\sigma}\right|
$$


for any $\sigma \in Z$ with $\left(\sigma^{\prime}, \sigma^{\prime \prime}\right) \neq(0,0)$. Moreover, we have

$$
\left|\psi_{i_{1} \ldots i_{p-1}}^{\sigma}\right| \leq \frac{\sqrt{m} M}{m_{0}}\left|\phi_{j(\sigma) i_{1} \ldots i_{p-1}}^{\sigma}\right|
$$

for any $\sigma \in Z$ with $\left(\sigma^{\prime}, \sigma^{\prime \prime}\right)=(0,0)$ by (8.2). Therefore, the series $\psi=\sum_{\sigma \in Z} \psi^{\sigma}$ converges by Lemma 5.1 .

If $Z=\mathbb{Z}^{n+m} \backslash\left\{\sigma_{0}\right\}$, then we have

$$
\phi=\Phi_{0} \wedge \psi+\bar{\partial}_{1} \psi+\phi^{\sigma_{0}} .
$$

Therefore, $\varphi=G \phi$ is $\bar{\partial}_{1}$-cohomologous to $G \phi^{\sigma_{0}}$. Since

$$
G \phi^{\sigma_{0}}=G \mathbf{e}\left(\left\langle\sigma_{0}, t^{\prime}\right\rangle+\sqrt{-1}\left\langle\sigma_{0}^{\prime \prime}, t^{\prime \prime}\right\rangle\right) \phi_{c}^{\sigma_{0}},
$$

we obtain

$$
H^{p}(X, \mathcal{O}(L)) \cong H^{p}(\mathbb{T}, \mathcal{O}) .
$$

(II) Suppose that the condition $(H)_{S}$ is not satisfied, but $H^{p}(X, \mathcal{O}(L))$ is a Hausdorff space for some $p$ with $1 \leq p \leq m$. Then $B_{\bar{\partial}_{1}}\left(X, \mathcal{F}^{0, p}(L)\right)$ is a closed subspace of $Z_{\bar{\partial}_{1}}\left(X, \mathcal{F}^{0, p}(L)\right)$. By Lemmas 8.1 and 8.2 the condition $(H)_{S}^{\prime \prime}$ is not satisfied. Then, for any $v \in \mathbb{N}$ there exists $\sigma(v)=\left(\sigma(v)^{\prime}, \sigma(v)^{\prime \prime}, \sigma(v)^{\prime \prime \prime}\right) \in Z$ with $\left(\sigma(v)^{\prime}, \sigma(v)^{\prime \prime}\right) \neq(0,0)$ such that

$$
\left\|K_{\sigma(v)}+d(L)\right\|<\frac{1}{v} \exp \left(-v\left|\sigma(v)^{\prime \prime}\right|\right) .
$$

We set

$$
\delta^{\sigma}:= \begin{cases}\frac{\exp \left(-v\left|\sigma(v)^{\prime \prime}\right|\right)}{\widetilde{K}_{\sigma(v), j(\sigma(v))}+\beta_{j(\sigma(v))}} & \text { if } \sigma=\sigma(v) \text { for some } v, \\ 0 & \text { otherwise. }\end{cases}
$$

For any $\sigma \in Z$ we take $i_{1}, \ldots, i_{p-1}$ with $1 \leq i_{1}<\cdots<i_{p-1} \leq m$ such that $j(\sigma) \notin$ $\left\{i_{1}, \ldots, i_{p-1}\right\}$, and we define

$$
\psi^{\sigma}:=\psi_{i_{1} \ldots i_{p-1}}^{\sigma} d \bar{z}_{i_{1}} \wedge \cdots \wedge d \bar{z}_{i_{p-1}},
$$

where

$$
\psi_{i_{1} \ldots i_{p-1}}^{\sigma}=\delta^{\sigma} \mathbf{e}\left(\left\langle\sigma, t^{\prime}\right\rangle+\sqrt{-1}\left\langle\sigma^{\prime \prime}, t^{\prime \prime}\right\rangle\right) .
$$

We consider the formal sum $\psi:=\sum_{\sigma \in Z} \psi^{\sigma}$. Since

$$
\frac{\exp \left(-v\left|\sigma(v)^{\prime \prime}\right|\right)}{\left|\widetilde{K}_{\sigma(v), j(\sigma(v))}+\beta_{j(\sigma(v))}\right|}>v,
$$

$\psi$ does not converge. On the other hand, we have

$$
\begin{aligned}
\Phi_{0} \wedge \psi^{\sigma}+\bar{\partial}_{1} \psi^{\sigma}= & \left(\widetilde{K}_{\sigma, j(\sigma)}+\beta_{j(\sigma)}\right) \psi_{i_{1} \ldots i_{p-1}}^{\sigma} d \bar{z}_{j(\sigma)} \wedge d \bar{z}_{i_{1}} \wedge \cdots \wedge d \bar{z}_{i_{p-1}} \\
& +\sum_{j \notin\left\{i_{1}, \ldots, i_{p-1}\right\}}\left(\widetilde{K}_{\sigma, j}+\beta_{j}\right) \psi_{i_{1} \ldots i_{p-1}}^{\sigma} d \bar{z}_{j} \wedge d \bar{z}_{i_{1}} \wedge \cdots \wedge d \bar{z}_{i_{p-1}} .
\end{aligned}
$$

Since

$$
\begin{aligned}
\left|\left(\widetilde{K}_{\sigma(v), j}+\beta_{j}\right) \delta^{\sigma(v)}\right| & \leq\left|\left(\widetilde{K}_{\sigma(v), j(\sigma(v))}+\beta_{j(\sigma(v))}\right) \delta^{\sigma(v)}\right| \\
& =\exp \left(-v\left|\sigma(v)^{\prime \prime}\right|\right),
\end{aligned}
$$


$\sum_{\sigma \in Z}\left(\Phi_{0} \wedge \psi^{\sigma}+\bar{\partial}_{1} \psi^{\sigma}\right)$ converges. By Lemma 9.2 there exists $\lambda \in H^{0}\left(X, \mathcal{F}^{0, p-1}\right)$ such that if we set

$$
\phi:=\sum_{\sigma \in Z}\left(\Phi_{0} \wedge \psi^{\sigma}+\bar{\partial}_{1} \psi^{\sigma}\right)
$$

then $\phi=\Phi_{0} \wedge \lambda+\bar{\partial}_{1} \lambda$. We express $\phi$ as in (7.4) and (7.5). We expand $\lambda$ as $\lambda=$ $\sum_{\sigma \in \mathbb{Z}^{n+m}} \lambda^{\sigma}$, where

$$
\begin{gathered}
\lambda^{\sigma}=\sum_{1 \leq i_{1}<\cdots<i_{p-1} \leq m} \lambda_{i_{1} \ldots i_{p-1}}^{\sigma} d \bar{z}_{i_{1}} \wedge \cdots \wedge d \bar{z}_{i_{p-1}}, \\
\lambda_{i_{1} \ldots i_{p-1}}^{\sigma}=b_{i_{1} \ldots i_{p-1}}^{\sigma} \mathbf{e}\left(\left\langle\sigma, t^{\prime}\right\rangle+\sqrt{-1}\left\langle\sigma^{\prime \prime}, t^{\prime \prime}\right\rangle\right), \quad b_{i_{1} \ldots i_{p-1}}^{\sigma} \in \mathbb{C} .
\end{gathered}
$$

For any $\sigma \in Z$ we have

$$
\Phi_{0} \wedge \psi^{\sigma}+\bar{\partial}_{1} \psi^{\sigma}=\Phi_{0} \wedge \lambda^{\sigma}+\bar{\partial}_{1} \lambda^{\sigma}
$$

We also have

$$
\begin{aligned}
\Phi_{0} \wedge \lambda^{\sigma}+\bar{\partial}_{1} \lambda^{\sigma}= & \sum_{1 \leq i_{1}<\cdots<i_{p-1} \leq m}\left\{\left(\widetilde{K}_{\sigma, j(\sigma)}+\beta_{j(\sigma)}\right) \lambda_{i_{1} \ldots i_{p-1}}^{\sigma} d \bar{z}_{j(\sigma)}\right. \\
& \left.+\sum_{j \notin\left\{j(\sigma), i_{1}, \ldots, i_{p-1}\right\}}\left(\widetilde{K}_{\sigma, j}+\beta_{j}\right) \lambda_{i_{1} \ldots i_{p-1}}^{\sigma} d \bar{z}_{j}\right\} \wedge d \bar{z}_{i_{1}} \wedge \cdots \wedge d \bar{z}_{i_{p-1}} .
\end{aligned}
$$

Comparing the coefficients of $d \bar{z}_{j(\sigma)} \wedge d \bar{z}_{i_{1}} \wedge \cdots \wedge d \bar{z}_{i_{p-1}}$ in both sides of (9.3), we obtain

$$
\begin{aligned}
& \left(\widetilde{K}_{\sigma, j(\sigma)}+\beta_{j(\sigma)}\right) \delta^{\sigma} \\
& \quad=\left(\widetilde{K}_{\sigma, j(\sigma)}+\beta_{j(\sigma)}\right) b_{i_{1} \ldots i_{p-1}}^{\sigma}+\sum_{k=1}^{p-1}(-1)^{k}\left(\widetilde{K}_{\sigma, i_{k}}+\beta_{i_{k}}\right) b_{i_{1} \ldots i_{k-1} j(\sigma) i_{k+1} \ldots i_{p-1}}^{\sigma} .
\end{aligned}
$$

Then we have

$$
\delta^{\sigma}=b_{i_{1} \ldots i_{p-1}}^{\sigma}+\sum_{k=1}^{p-1}(-1)^{k} \frac{\widetilde{K}_{\sigma, i_{k}}+\beta_{i_{k}}}{\widetilde{K}_{\sigma, j(\sigma)}+\beta_{j(\sigma)}} b_{i_{1} \ldots i_{k-1} j(\sigma) i_{k+1} \ldots i_{p-1}}^{\sigma} .
$$

Since

$$
\left|\frac{\widetilde{K}_{\sigma, i_{k}}+\beta_{i_{k}}}{\widetilde{K}_{\sigma, j(\sigma)}+\beta_{j(\sigma)}}\right| \leq 1
$$

and $\sum_{\sigma \in \mathbb{Z}^{n+m}} \lambda^{\sigma}$ converges, $\sum_{\sigma \in Z} \psi^{\sigma}$ must converge. This is a contradiction.

\section{Examples}

In this section we give examples which show that each of the cases in Theorem 9.1 really occurs.

Example 10.1. Let $\Gamma$ be a discrete subgroup of rank 3 in $\mathbb{C}^{2}$ whose period matrix is

$$
P=\left(\begin{array}{lll}
1 & 0 & \sqrt{-1} \alpha \\
0 & 1 & \sqrt{-1}
\end{array}\right), \quad \alpha \in \mathbb{R} \backslash \mathbb{Q} .
$$


Then $X=\mathbb{C}^{2} / \Gamma$ is a toroidal group since the irrationality condition (IS) is fulfilled. Let $z=\left(z_{1}, z_{2}\right)$ be standard coordinates of $\mathbb{C}^{2}$ with respect to $P$. We write $P=\left(e_{1}, e_{2}, s_{1}\right)$, where $s_{1}={ }^{\mathrm{t}}\left(s_{11}, s_{21}\right)={ }^{\mathrm{t}}(\sqrt{-1} \alpha, \sqrt{-1})$. We set $s_{2}={ }^{\mathrm{t}}\left(s_{12}, s_{22}\right):={ }^{\mathrm{t}}(0, \sqrt{-1})$. Let $t=$ $\left(t_{1}, t_{2}, t_{3}, t_{4}\right)$ be real coordinates of $\mathbb{C}^{2}$ with respect to $\left\{e_{1}, e_{2}, s_{1}, s_{2}\right\}$. In this case the matrices $A, B$ and $C$ in Section 2 are as follows:

$$
A=\left(\operatorname{Re}\left(s_{i j}\right)\right)=0, \quad B=\left(\operatorname{Im}\left(s_{i j}\right)\right)=\left(\begin{array}{cc}
\alpha & 0 \\
1 & 1
\end{array}\right) \quad \text { and } \quad C=\left(\begin{array}{cc}
\frac{1}{\alpha} & 0 \\
-\frac{1}{\alpha} & 1
\end{array}\right) .
$$

For any $\sigma=\left(\sigma_{1}, \sigma_{2}, \sigma_{3}\right) \in \mathbb{Z}^{3}$ we have

$$
K_{\sigma, 1}=\sqrt{-1}\left(\sigma_{1} \alpha+\sigma_{2}\right)-\sigma_{3} .
$$

Furthermore, we have $K_{\sigma}=K_{\sigma, 1}$ and $\widetilde{K}_{\sigma}=\widetilde{K}_{\sigma, 1}=\pi K_{\sigma} \alpha$.

We consider a homomorphism $d: \Gamma \longrightarrow \mathbb{R}$ such that $d\left(e_{1}\right)=d\left(e_{2}\right)=0$ and $d\left(s_{1}\right) \in$ $\mathbb{R} \backslash \mathbb{Z}$. Let $L$ be the homogeneous line bundle over $X$ given by a representation $\rho(\gamma)=$ $\mathbf{e}(d(\gamma))(\gamma \in \Gamma)$. In this case we have $\alpha_{1}=-\sqrt{-1}\left(d\left(s_{1}\right) / \alpha\right), \beta_{1}=-\left(\pi d\left(s_{1}\right)\right) / \alpha$ and $d(L)=$ $-d\left(s_{1}\right)$. Then we obtain

$$
K_{\sigma}+d(L)=\sqrt{-1}\left(\sigma_{1} \alpha+\sigma_{2}\right)-\left(\sigma_{3}+d\left(s_{1}\right)\right) .
$$

Since the equality $\widetilde{K}_{\sigma}+\beta_{1}=0$ is equivalent to $K_{\sigma}+d(L)=0$, we have $Z=\mathbb{Z}^{3}$. We note that

$$
\left|K_{\sigma}+d(L)\right| \geq\left|\sigma_{1} \alpha+\sigma_{2}\right| .
$$

We assume that $\alpha$ is an algebraic number. By Liouville's theorem we see at once that the condition $(H)_{S}$ is satisfied. This is an example for the case (i) in (I) in Theorem 9.1.

Example 10.2. We consider a period matrix

$$
P=\left(\begin{array}{ccc}
1 & 0 & \sqrt{-1} \\
0 & 1 & \alpha
\end{array}\right), \quad \alpha \in \mathbb{R} \backslash \mathbb{Q} .
$$

It is easily seen that $X=\mathbb{C}^{2} / \Gamma$ is a toroidal group, where $\Gamma$ is the discrete subgroup of $\mathbb{C}^{2}$ given by $P$. As in Example 10.1 , we write $P=\left(e_{1}, e_{2}, s_{1}\right)$. Take a homomorphism $d: \Gamma \longrightarrow \mathbb{R}$ such that $d\left(e_{1}\right)=d\left(e_{2}\right)=0$ and $d\left(s_{1}\right) \in \mathbb{R} \backslash \mathbb{Z}$. Then a representation $\rho=\mathbf{e}(d)$ of $\Gamma$ defines a homogeneous line bundle $L$ over $X$. We obtain

$$
K_{\sigma}+d(L)=\sqrt{-1} \sigma_{1}+\sigma_{2} \alpha-\sigma_{3}-d\left(s_{1}\right)
$$

by a straightforward calculation.

We assume that $\alpha$ is an algebraic number. We set $d\left(s_{1}\right):=\alpha$. By Liouville's theorem there exist $C>0$ and $N \in \mathbb{N}$ such that

$$
|q \alpha-p| \geq \frac{C}{|q|^{N}}
$$

for $p, q \in \mathbb{Z}$ with $q \neq 0$. Then we obtain

$$
\begin{aligned}
\left|K_{\sigma}+d(L)\right| & \geq\left|\left(\sigma_{2}-1\right) \alpha-\sigma_{3}\right| \\
& \geq \frac{C}{\left|\sigma_{2}-1\right|^{N}} \\
& \geq C e^{-N} \exp \left(-N\left|\sigma_{2}\right|\right)
\end{aligned}
$$


for any $\sigma \in \mathbb{Z}^{3}$ with $\sigma_{2} \neq 1$. This is the condition $(H)_{S}^{\prime \prime}$. Moreover, for $\sigma_{0}=(0,1,0)$ we have

$$
\widetilde{K}_{\sigma_{0}}+\beta_{1}=\pi\left(K_{\sigma_{0}}+d(L)\right)=0 .
$$

Example 10.3. The following example is a modification of Vogt's example [10]. In Example 10.2 we set

$$
\alpha=d\left(s_{1}\right):=\sum_{j=1}^{\infty} 10^{\left(-10^{j !}\right)}
$$

For any $v \in \mathbb{N}$ we define

$$
q_{\nu}:=10^{\nu !} 10^{10^{\nu !}} \text { and } \quad p_{v}:=10^{\nu !} 10^{10^{\nu !}}\left(\sum_{j=1}^{\nu} 10^{\left(-10^{j !}\right)}\right) .
$$

Then there exists $C>0$ such that

$$
\left|q_{\nu} \alpha-p_{\nu}\right| \leq C \exp \left(-q_{\nu}^{2}\right)
$$

for sufficiently large $v$. We set

$$
\sigma^{(v)}=\left(\sigma_{1}^{(v)}, \sigma_{2}^{(v)}, \sigma_{3}^{(v)}\right):=\left(0, q_{v}+1, p_{v}\right)
$$

for $v \in \mathbb{N}$. We suppose that the condition $(H)_{S}$ is satisfied. By Lemmas 8.1 and 8.2 we can take constants $\widetilde{C}>0$ and $\widetilde{a} \geq 0$ such that

$$
\left|K_{\sigma}+d(L)\right| \geq \widetilde{C} \exp \left(-\widetilde{a}\left|\sigma_{2}\right|\right)
$$

for $\sigma \in Z$ with $\left(\sigma_{1}, \sigma_{2}\right) \neq(0,0)$. Especially, the above inequality holds for $\sigma^{(\nu)}$. On the other hand, we have

$$
\left|K_{\sigma^{(\nu)}}+d(L)\right|=\left|\sigma_{2}^{(\nu)} \alpha-\sigma_{3}^{(\nu)}-\alpha\right|=\left|q_{\nu} \alpha-p_{\nu}\right| .
$$

Then we obtain

$$
0<\frac{\widetilde{C}}{C} \leq \exp \left(-q_{v}^{2}+\widetilde{a}\left(q_{v}+1\right)\right)
$$

for sufficiently large $v$, which is impossible. Thus we conclude that the condition $(H)_{S}$ is not satisfied.

\section{REFERENCES}

[1] Y. Abe. Homogeneous line bundles over a toroidal group. Nagoya Math. J. 116 (1989), 17-24.

[2] Y. Abe. ${ }^{o} K_{0}$-quasi-abelian varieties with complex multiplication. Forum Math. 25 (2013), 677-702.

[3] Y. Abe. Quasi-abelian varieties given by certain algebraic number fields. Toyama Math. J. 36 (2013-2014), 27-36.

[4] Y. Abe and K. Kopfermann. Toroidal Groups (Lecture Notes in Mathematics, 1759). Springer, Berlin, 2001.

[5] H. Kazama. $\bar{\partial}$-Cohomology of $(H, C)$-groups. Publ. RIMS, Kyoto Univ. 20 (1984), 297-317.

[6] H. Kazama and T. Umeno. Dolbeault isomorphisms for holomorphic vector bundles over holomorphic fiber spaces and applications. J. Math. Soc. Japan 45 (1993), 121-130.

[7] K. Kopfermann. Maximale Untergruppen abelscher komplexer Liescher gruppen. Schr. Math. Inst. Univ. Münster 29 (1964).

[8] A. Morimoto. Non-compact complex Lie groups without non-constant holomorphic functions. Proc. Conf. Complex Analysis (Minneapolis, 1964). Springer, Berlin, 1965, pp. 256-272. 
[9] A. Morimoto. On the classification of non-compact complex abelian Lie groups. Trans. Amer. Math. Soc. 123 (1966), 200-228.

[10] Ch. Vogt. Line bundles on toroidal groups. J. Reine Angew. Math. 335 (1982), 197-215.

\section{Yukitaka Abe}

Graduate School of Science and Engineering for Research University of Toyama

Toyama 930-8555

Japan

(E-mail:abe@sci.u-toyama.ac.jp) 